Received: 2016.09.12 Accepted: 2016.10.14 Published: 2017.03.21

\title{
Primary Pulmonary Diffuse Large B Cell Non-Hodgkin's Lymphoma: A Case Report and Literature Review
}

Authors' Contribution:

Study Design A

Data Collection B

Statistical Analysis C

Data Interpretation D

Manuscript Preparation E

Literature Search F

Funds Collection G
BCDEF 1 Ziqiang Zhu

DEF 1 Wei Liu

EF 1 Omar Mamlouk

BD 2 James E. O'Donnell

BCDE 1 Debabrata Sen

ABCDEF 3 Boris Avezbakiyev
1 Department of Medicine, The Brookdale University Hospital and Medical Center, Brooklyn, NY, U.S.A.

2 Department of Pathology, The Brookdale University Hospital and Medical Center, Brooklyn, NY, U.S.A.

3 Division of Hematology/Oncology, The Brookdale University Hospital and Medical Center, Brooklyn, NY, U.S.A.
Corresponding Author: Conflict of interest:

None declared

\begin{tabular}{|c|c|}
\hline Patient: & Female, 48 \\
\hline Final Diagnosis: & Primary pulmonary DLBCL \\
\hline Symptoms: & Cough $\bullet$ weigh loss \\
\hline Medication: & R-CHOP (rituximab, cyclophosphamide, doxorubicin, vincristine, and prednisone) \\
\hline Clinical Procedure: & Bone marrow biopsy • CT-guided lung biopsy \\
\hline Specialty: & Oncology \\
\hline Objective: & Rare disease \\
\hline Background: & $\begin{array}{l}\text { Primary pulmonary diffuse large B cell lymphoma (DLBCL) is extremely rare neoplasm representing only } 0.5-1 \% \\
\text { of primary pulmonary malignancies. These patients usually have non-specific clinical presentation and radio- } \\
\text { logical findings. Therefore, it is important to increase awareness of this rare disease, as the correct character- } \\
\text { ization of the tumors will have therapeutic and prognostic implications. }\end{array}$ \\
\hline Case Report: & $\begin{array}{l}\text { We present the case of a middle-aged Hispanic woman with chronic cough and an abnormal chest X-ray reveal- } \\
\text { ing a lung mass, who was found to have primary pulmonary DLBCL. She underwent } 6 \text { cycles of R-CHOP (ritux- } \\
\text { imab, cyclophosphamide, doxorubicin, vincristine, and prednisone) therapy and attained complete remission. }\end{array}$ \\
\hline Conclusions: & $\begin{array}{l}\text { With its non-specific presentation, the diagnosis of primary pulmonary DLBCL is very challenging and often } \\
\text { leads to misdiagnosis or delayed diagnosis. The pathogenesis of primary pulmonary DLBCL is still poorly under- } \\
\text { stood. The choice of treatment approach should be based on the biological characteristic of the tumor, stage, } \\
\text { and performance status. }\end{array}$ \\
\hline
\end{tabular}

MeSH Keywords: $\quad$ Lung Neoplasms • Lymphoma, B-Cell • Solitary Pulmonary Nodule

Full-text PDF: http://www.amjcaserep.com/abstract/index/idArt/901528

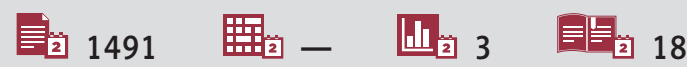




\section{Background}

Primary pulmonary lymphoma is defined as clonal lymphoid infiltration of the lung with or without hilar lymph nodes involvement at the time of diagnosis or up to 3 months thereafter [1]. It is a rare neoplasm that represents only $0.5-1 \%$ of primary pulmonary malignancies [2]. Most (70-90\%) primary pulmonary lymphomas are low-grade mucosa-associated lymphoid tissue (MALT) type, and only about $10 \%$ are primary diffuse large $B$ cell lymphomas (DLBCL) [3]. Therefore, primary DLBCL of the lung is extremely rare. It is generally found in elderly or immunosuppressed patients [4,5]. Clinically, these patients usually present with non-specific symptoms. Radiologically, it is extremely difficult to differentiate from more common lung malignancies such as bronchogenic carcinoma. Therefore, it is important to increase awareness of this rare disease, as the correct characterization of the tumors has therapeutic and prognostic implications. We present the case of a middle-aged

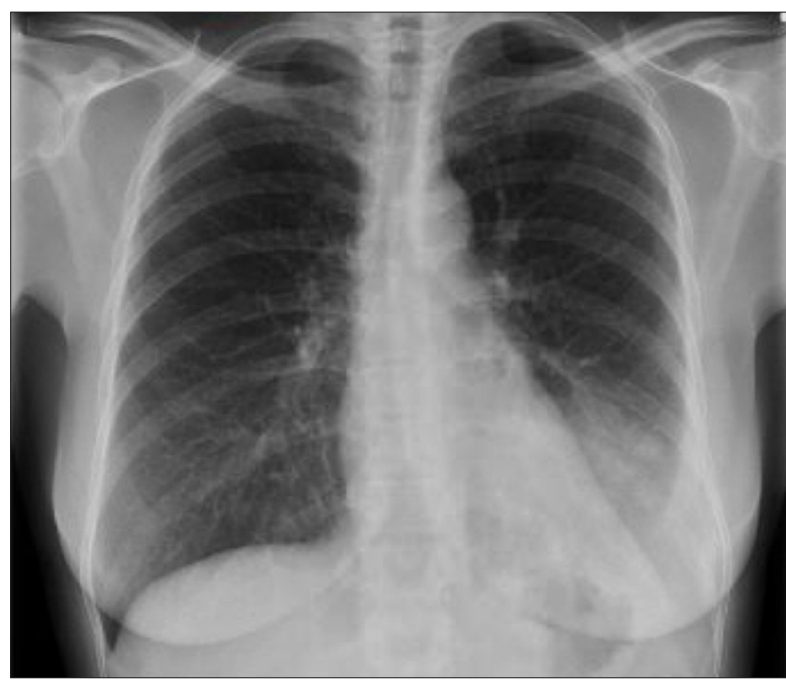

Figure 1. CXR shows large left lower lobe infiltrate. woman with chronic cough and an abnormal chest X-ray revealing a lung mass, who was found to have primary pulmonary DLBCL. We further discuss the current understanding of primary pulmonary $\mathrm{DLBCL}$, including possible pathogenesis and treatment options.

\section{Case Report}

A 48-year old Hispanic woman presented with 2-month history of non-productive cough, associated with night sweats and 10-pound weight loss. She denied any hemoptysis. She was an active smoker who had smoked an average of half a pack a day for the past 35 years. The physical examination was significant for decreased breath sound on the left lower base, but the rest of her physical examination was unremarkable. Laboratory investigation revealed WBC $14.7 \times 10^{9} / \mathrm{L}, \mathrm{Hb}$ $11.0 \mathrm{~g} / \mathrm{dL}$, LDH 614 IU/L (normal range 313-614 IU/L), normal LFT and renal function, and negative HIV results. She underwent radiological evaluation with chest $\mathrm{X}$-ray, which revealed a large left lower lobe infiltrate (Figure 1). This was followed by a CT scan of the chest (Figure 2A), which showed a $5.5-\mathrm{cm}$ left lower lobe lung mass and narrowing of the left lower lobe bronchus suspicious for carcinoma. Considering her symptoms, including chronic cough, and significant weight loss, as well as respiratory findings and history of smoking, malignancy remained high in the differential diagnosis. To further proceed with the diagnosis, she underwent a CT-guided core biopsy of a large left lower lobe lung mass. Histopathological staining of the tissue revealed proliferation of large cells (Figure $3 \mathrm{~A}, 3 \mathrm{~B}$ ) positive for CD20, CD43, Bcl-2, Bcl-6, and MUM-1 (focally) and negative for CD10 and Cyclin D1 (Figure 3C-3E). The proliferation marker, Ki67 (Figure 3F), was highly positive (60\%), indicating a high-grade neoplasm. Therefore, it is most consistent with a diagnosis of DLBCL of the lung. The concomitant CT scan of the abdomen and pelvis did not show any evidence of

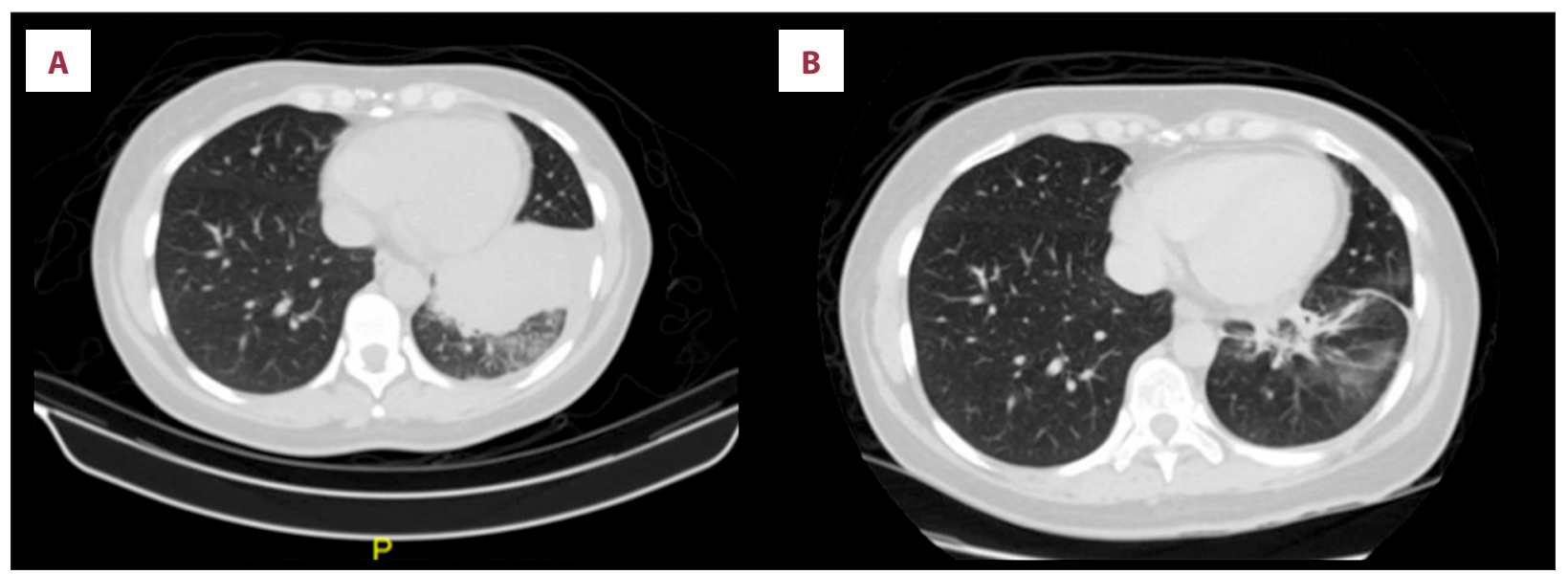

Figure 2. (A) CT scan of the chest shows a $5.5 \mathrm{~cm}$ left lower lobe lung mass and narrowing of the left lower lobe bronchus. (B) Repeat CT scan of the chest after 4 cycles of R-CHOP therapy. 

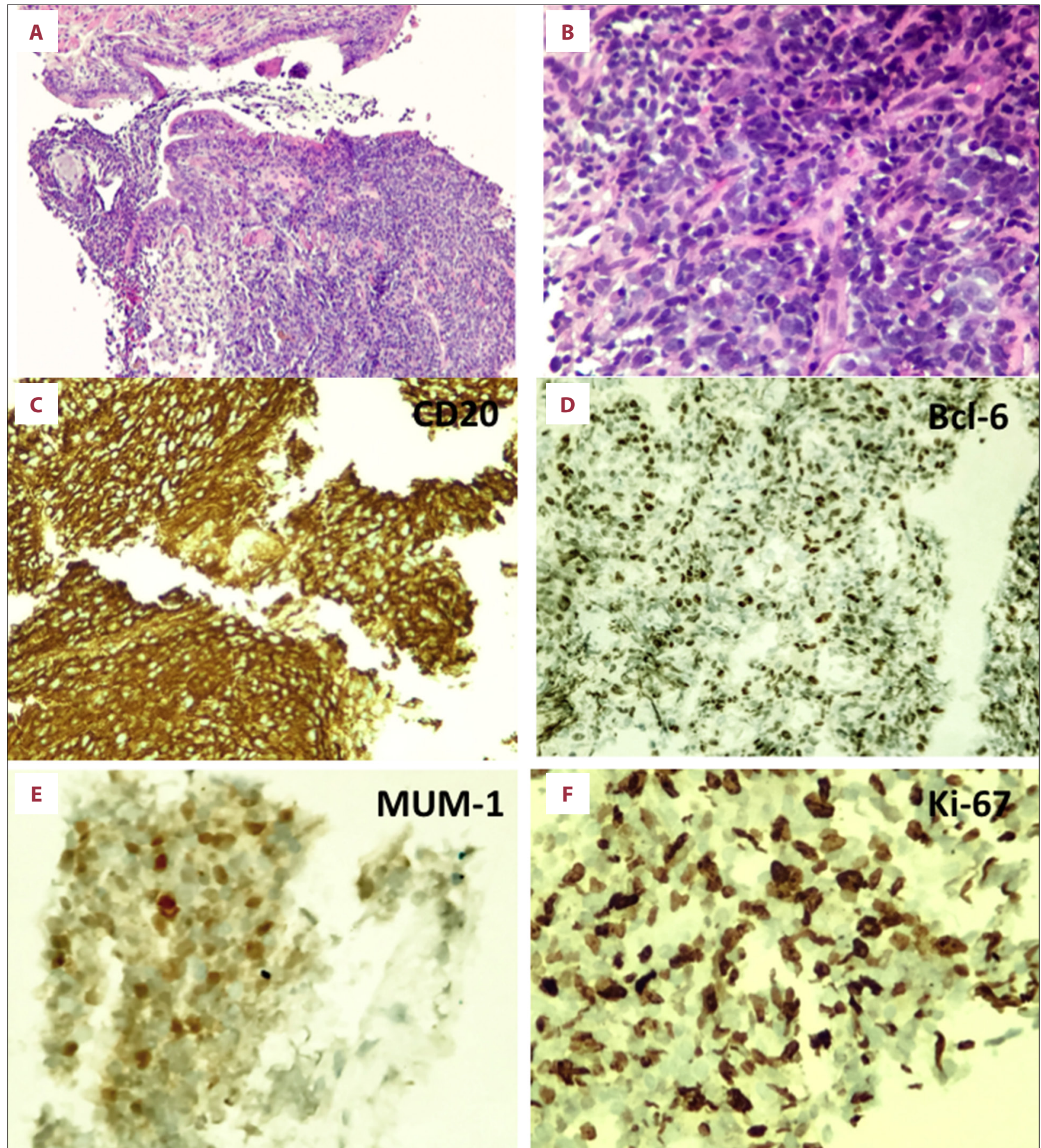

Figure 3. Hematoxylin and eosin staining of the lung biopsy demonstrate diffuse proliferation of large cells at lower power (10x, A) and high power $(100 \times, B)$. Immunohistochemistry staining (C-F) of the lung biopsy shows positive for CD20, Bcl-6, MUM-1, and Ki67.

extrathoracic involvement. A positron emission tomographycomputed tomography $(\mathrm{PET} / \mathrm{CT})$ scan showed a $6.7 \times 6 \mathrm{~cm}$ retrocardiac mass in the lower lobe of the left lung, extending from left hilar region with SUV of 17.3, as well as diffuse FDG uptake throughout the marrow of the axial and proximal appendicular skeleton. Further bone marrow biopsy revealed no lymphoma involvement. Therefore, she was diagnosed with DLBCL stage IE. The Revised International Prognostic Index (RIPI) score at the time of diagnosis was 0 . She was followed up in the oncology clinic and finished 6 cycles of R-CHOP (rituximab, cyclophosphamide, doxorubicin, vincristine, and prednisone) therapy. She tolerated the regimen well without any 
significant complications and attained complete remission as determined by negative PET and CT scans (Figure 2B) performed after the 4th cycle. The patient remains in remission both clinically and radiologically 2 years later.

\section{Discussion}

Primary pulmonary DLBCL is a rare disease, mostly affecting immunocompromised or elderly individuals. It was reported as the second most common sub-type of primary pulmonary lymphoma, accounting for $11-19 \%$ of primary pulmonary lymphomas [1]. However, due to its rapid spread into the mediastinum and extrathoracic locations, the true incidence of primary DLBCL of lung may be underestimated. With its non-specific presentation, the diagnosis of primary pulmonary DLBCL is very challenging and often leads to misdiagnosis or delayed diagnosis.

The pathogenesis of primary pulmonary DLBCL is still poorly understood. Studies have shown that pulmonary DLBCL can arise de novo; however, it may more commonly be transformed from primary pulmonary marginal zone B cell lymphoma of mucosa-associated lymphoid tissue (MALT). MALT lymphoma is believed to arise as a result of chronic inflammation of the marginal zone B cells of the "bronchial-associated lymphoid tissue". In contrast, pulmonary DLBCL is much more aggressive and tends to manifest with systemic "B" symptoms, including loss of more than $10 \%$ of body weight over a 6 -month period [1]. MALT lymphoma is low-grade B cell lymphoma that is rarely reported to progress to a high-grade tumor. Almost $50 \%$ of primary pulmonary DLBCLs coexist with pulmonary MALT lymphomas. Because of these mixed and transitional forms, it has been proposed to progress from pulmonary MALT lymphoma to DLBCL of the lungs. In addition, transformation from MALT lymphoma to DLBCL has also been reported in patients with breast MALT lymphoma [6]. However, due to extreme rarity of the disease, very little evidence is available regarding the mechanism by which pulmonary MALT lymphoma progresses to DLBCL. Since gastric MALT lymphoma is relatively common, extensive research has been done to investigate the progression of gastric MALT to aggressive lymphomas. Based on the similar heterogeneous feature in gastric and pulmonary MALT lymphoma, one can speculate that both entities share some of the common pathways of progression to a more aggressive form of DLBCL. Studies from gastric MALT lymphoma have suggested that high-grade lymphomas arise through blastic transformation of a low-grade preexisting component, based on the observations that gastric MALT lymphoma is a genetically heterogeneous disease with immunohistochemical evidence of both low- and high-grade clones expressing the same class of immunoglobulin light chains with identical light chain restriction [7-9]. Regression of low-grade gastric MALT lymphoma is very common after eradication of $H$. pylori, but high-grade components may progress [10]. However, no particular microorganism has been linked to the development of primary MALT lymphoma of the lungs. In addition, studies have revealed that up to $60 \%$ of gastric MALT lymphomas bear chromosomal aberrations $t(11 ; 18)$ translocation, but it was not detected in DLBCL [11]. Therefore, $t(11 ; 18)$-positive MALT lymphoma is very unlikely to transform into high-grade lymphomas. The fact that $\mathrm{t}(11 ; 18)$-negative cases share certain aberrations such as 3q26.2-27, 6q23.3-25, 7q31, 11q2324 , and $18 q 21$ between MALT and DLBCL suggests that this group of tumors may eventually progress to $\operatorname{DLBCL}[11,12]$. Another gene, c-Myc, has also been reported to predict aggressive transformation [13]. Overexpression of c-Myc can repress microRNA-34a, which eventually promotes high-grade transformation [14]. Unfortunately, the relationship between primary pulmonary MALT lymphoma and primary pulmonary $\mathrm{DLBCL}$ is less clear and warrants further research.

MALT lymphoma is a very indolent disease and has the potential for spontaneous regression [3], while DLBCL is much more aggressive and need aggressive treatment even in the early stage. However, due to its rarity, no randomized clinical trial has been performed to establish the optimal treatment option. Different therapeutic modalities have been used in clinical practice, including watchful waiting, surgery, chemotherapy, or chemotherapy followed by radiotherapy $[3,15]$. The choice of treatment approach should be based on the biological characteristic of the tumor, stage, and performance status. Surgery may be considered in the localized form of primary pulmonary DLBCL [15]. Radiation therapy has a limited role in pulmonary DLBCL, probably due to its toxicity in the lungs. Systemic chemotherapy with $\mathrm{CHOP}$ has resulted in significant clinical and radiological response in pulmonary $D L B C L$. The addition of rituximab, a monoclonal antibody against $C D 20$ found on the surface of B cells, to CHOP has been shown to improve overall survival in DLBCL with nodal lymphoma and in some extranodal types $[16,17]$. The $\mathrm{R}-\mathrm{CHOP}$ regimen is considered the criterion standard in DLBCL with nodal presentation. In clinical practice, despite lack of solid evidence, R-CHOP has been used as the standard therapy in patients with primary pulmonary DLBCL for the past 5 years. However, Aviles et al. [18], in an open-label study in 42 patients with primary $D L B C L$ of the lungs, failed to show any improvement of outcome with the addition of rituximab to CHOP. The authors agreed that a controlled clinical trial is the best way to study the efficacy of $\mathrm{R}-\mathrm{CHOP}$ regimen in primary pulmonary $\mathrm{DLBCL}$; however, due to its rarity, it is very difficult to recruit a sufficient number of patients to achieve any statistical significance. Therefore, they recommended that the decision to add rituximab to $\mathrm{CHOP}$ in the treatment of primary pulmonary $D L B C L$ should be made on an individual basis. 


\section{Conclusions}

With its non-specific presentation, the diagnosis of primary pulmonary $D L B C L$ is very challenging and often leads to misdiagnosis or delayed diagnosis. The pathogenesis of primary pulmonary DLBCL is still poorly understood. The choice of treatment approach should be based on the biological characteristic of the tumor, stage, and performance status.

\section{References:}

1. Piña-Oviedo S, Weissferdt A, Kalhor N, Moran CA: Primary pulmonary lymphomas. Adv Anat Pathol, 2015; 22(6): 355-75

2. Chilosi M, Zinzani PL, Poletti V: Lymphoproliferative lung disorders. Semin Respir Crit Care Med, 2005; 26: 490-501

3. Zinzani PL, Martelli M, Poletti V et al: Practice guidelines for the management of extranodal non-Hodgkin's lymphomas of adult non-immunodeficient patients. Part I: primary lung and mediastinal lymphomas. A project of the Italian Society of Hematology, the Italian Society of Experimental Hematology and the Italian Group for Bone Marrow Transplantation. Haematologica, 2008; 93: 1364-71

4. Shahani L, McKenna M: Primary pulmonary lymphoma in a patient with advanced AIDS. BMJ Case Rep, 2014; 2014. pii: bcr2013203265

5. Seeram V, Shujaat A, Jones L, Bajwa A: An 82-year-old woman with left upper lobe atelectasis. Diffuse large B-cell lymphoma. Chest, 2012; 142(6): 1669-74

6. Arslan SH, Uyeturk U, Tekgunduz E et al: Primary breast mucosa-associated lymphoid tissue (malt) lymphoma transformation to diffuse large B-cell lymphoma: A case report. Turk J Haematol, 2012; 29: 274-77

7. Jung JH, Jung HY, Yoon $\mathrm{H}$ et al: Two cases of diffuse large b-cell lymphomas in the cervical lymph nodes in patients with low-grade gastric marginal zone B-cell lymphoma (MALT Lymphoma). Clin Endosc, 2013; 46: 288-92

8. Maeshima AM, Taniguchi $\mathrm{H}$, Toyoda $\mathrm{K}$ et al: Clinicopathological features of histological transformation from extranodal marginal zone B-cell lymphoma of mucosa-associated lymphoid tissue to diffuse large B-cell lymphoma: An analysis of 467 patients. Br J Haematol, 2016;1 74(6): 923-31

9. Machado I, Lavernia J, Illueca C et al: Histologic transformation to diffuse large B cell lymphoma with profuse signet-ring cell change in bone marrow and lymph node biopsies in a patient with marginal zone lymphoma. A cytologic-histologic correlation. Diagn Cytopathol, 2016; 44(10): 860-63

10. Iwano M, Watanabe N, Matsushima Y et al: Rapid development of diffuse large B-Cell lymphoma after successful eradication of Helicobacter pylori for gastric MALT lymphoma. Am J Gastroenterol, 2006; 101: 2878-83

\section{Acknowledgements}

We thank the excellent support provided by the medical staff at the Brookdale University Hospital and Medical Center.

11. Schreuder MI, Hoeve MA, Hebeda KM et al: Mutual exclusion of $t(11 ; 18)$ (q21;q21) and numerical chromosomal aberrations in the development of different types of primary gastric lymphomas. Br J Haematol, 2003; 123 590-99

12. Starostik P, Patzner J, Greiner A et al: Gastric marginal zone B-cell lymphomas of MALT type develop along 2 distinct pathogenetic pathways. Blood 2002; 99(1): 3-9

13. Huang W, Guo L, Liu H et al: C-MYC overexpression predicts aggressive transformation and a poor outcome in mucosa-associated lymphoid tissue lymphomas. Int J Clin Exp Pathol, 2014; 7: 5634-44

14. Craig VJ, Cogliatti SB, Imig J et al: Myc-mediated repression of microRNA34a promotes high-grade transformation of B-cell lymphoma by dysregulation of FoxP1. Blood, 2011; 117: 6227-36

15. Vanden Eynden F, Fadel E, de Perrot $M$ et al: Role of surgery in the treatment of primary pulmonary B-cell lymphoma. Ann Thorac Surg, 2007; 83(1): 236-40

16. Pfreundschuh M, Trümper L, Osterborg A et al: CHOP-like chemotherapy plus rituximab versus $\mathrm{CHOP}$ like chemotherapy alone in young patients with good-prognosis diffuse large-B-cell lymphoma: A randomised controlled trial by the MabThera International Trial (MInT) Group. Lancet Oncol, 2006; 7(5): 379-91

17. Coiffier B, Thieblemont C, Van Den Neste E et al: Long-term outcome of patients in the LNH-98.5 trial, the first randomized study comparing rituximab-CHOP to standard CHOP chemotherapy in DLBCL patients: A study by the Groupe d'Etudes des Lymphomes de l'Adulte. Blood, 2010; 116(12): 2040-45

18. Aviles A, Nambo MJ, Huerta-Guzman J et al: Rituximab in the treatment of diffuse large B-cell lymphoma primary of the lung. Hematology, 2013; 18 81-84 